\title{
TRATAMIENTO DE AGUAS RESIDUALES DE UNA INDUSTRIA PROCESADORA DE PESCADO EN REACTORES ANAERÓBICOS DISCONTINUOS
}

\section{WASTEWATER TREATMENT OF A FISH PROCESSING INDUSTRY IN BATCH ANAEROBIC REACTORS}

\begin{abstract}
Julio César Marín Leal', Carlos Aníbal Chinga Panta², Abrahan Isaac Velásquez Ferrín³, Pierre Andrés González Cabo4, Luz María Zambrano Rodríguez ${ }^{5}$
\end{abstract}

Fecha de recepción: 21 de Julio de 2014

Fecha de aprobación: 13 de Febrero de 2015

Referencia: J.C. Marín Leal, C.A. Chinga Panta, A.I. Velásquez Ferrín, P.A. González Cabo, L.M. Zambrano Rodríguez. (2015). Tratamiento de aguas residuales de una industria procesadora de pescado en reactores anaeróbicos discontinuos. Ciencia e Ingeniería Neogranadina, 25 (1), pp. 27 - 42

\section{RESUMEN}

En el presente trabajo se evaluó el tratamiento de las aguas residuales de una industria procesadora de pescado de la ciudad de Manta (Ecuador), en reactores anaeróbicos discontinuos, y se estableció su adecuación a las normas ambientales vigentes en materia de vertido. Para ello, se realizaron ensayos de laboratorio en reactores discontinuos de $1 \mathrm{~L}$, con un tiempo de contacto de $24 \mathrm{~h}$ y provistos de un lodo anaerobio procedente de una planta de tratamiento de aguas residuales domésticas. Dicho efluente fue diluido con agua destilada en proporciones de $33 \%, 66 \%$ y $100 \%$, correspondientes a las etapas I, II y III, respectivamente. Durante cada etapa se monitorearon los

1. Biólogo, M.Sc., Ph.D., Investigador del Programa Prometeo. Departamento Central de Investigación (DCI), Universidad Laica "Eloy Alfaro" de Manabí (ULEAM). Manta, Provincia de Manabí, Ecuador. Profesor Titular, Facultad de Ingeniería. Escuela de Ingeniería Civil, Departamento de Ingeniería Sanitaria y Ambiental (DISA). Universidad del Zulia, Maracaibo, Venezuela, jmarin@fing.luz.edu.ve

2. Biólogo Marino, M.Sc., Investigador II. Departamento Central de Investigación (DCI). Universidad Laica "Eloy Alfaro" de Manabí (ULEAM). Manta, Provincia de Manabí, Ecuador, carlos.chinga@uleam.edu.ec

3. Biólogo Marino, M.Sc., Docente-Investigador, Departamento Central de Investigación (DCI). Universidad Laica "Eloy Alfaro" de Manabí (ULEAM), Manta, Provincia de Manabí, Ecuador, abrahan.velasquez@uleam.edu.ec

4. Auxiliar de Investigación, Estudiante de Ingeniería de Recursos Naturales y Ambiente, Departamento Central de Investigación (DCI). Universidad Laica "Eloy Alfaro" de Manabí (ULEAM), Manta, Provincia de Manabí, Ecuador, andres-pag@hotmail.com

5. Auxiliar de Investigación, Estudiante de Ingeniería de Recursos Naturales y Ambiente, Departamento Central de Investigación (DCI). Universidad Laica "Eloy Alfaro" de Manabí (ULEAM), Manta, Provincia de Manabí, Ecuador, lucy_zambrano@hotmail.es 
siguientes parámetros, de acuerdo con los métodos estándares: $\mathrm{pH}$, alcalinidad total, $\mathrm{DBO}_{5.20^{\prime}}$ DQO, nitrito, amonio, nitrógeno total Kjeldahl (NTK), ortofostato, sulfato, sólidos suspendidos totales (SST) y sólidos suspendidos volátiles (SSV). Los resultados muestran porcentajes de

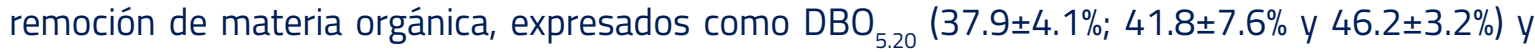
DQO (34.7 $\pm 9.7 \% ; 36.9 \pm 9.2 \%$ y $43.8 \pm 4.1 \%$, para las etapas I, II y III, respectivamente), relativamente bajos como resultado del origen del inóculo usado, así como del contenido relativo de sales en el efluente industrial. Las remociones de amonio, NTK y ortofosfato estuvieron entre 60-95\%, 25$37 \%$ y $6-25 \%$, respectivamente. Bajo las condiciones de los ensayos realizados, el efluente tratado requiriere de la aplicación de un postratamiento para reducir el contenido de materia orgánica y nutrientes a los límites permisibles de descarga establecidos en la República de Ecuador.

Palabras clave: Efluente industrial, materia biodegradable, lodo anaerobio, reactores discontinuos, tratabilidad anaeróbica.

\section{ABSTRACT}

In this paper, the treatment of wastewater from a fish processing industry (Manta, Ecuador) in batch anaerobic reactors was evaluated and its adjustment to current environmental standards for discharge was established. For this purpose, laboratory tests were performed in $1 \mathrm{~L}$ reactors supplied with sludge from an anaerobic treatment plant of domestic wastewater, with a contact time of 24 h. The effluent was diluted with distilled water in proportions of $33 \%, 66 \%$, and $100 \%$ corresponding to the stages I, II, and III respectively. During each stage, the following parameters were monitored according to standard methods: $\mathrm{pH}$, total alkalinity, $\mathrm{BOD}_{5.20^{\prime}} \mathrm{COD}$, nitrite, ammonium, total Kjeldahl nitrogen (TKN), orthophosphate, sulfate, total suspended solids (TSS) and volatile suspended solids (VSS). Results show removal percentages of organic matter, expressed as $\mathrm{BOD}_{5.20}(37.9 \pm 4.1 \%$, $41.8 \pm 7.6 \%$, and $46.2 \pm 3.2 \%)$ and COD $(34.7 \pm 97 \%, 36.9 \pm 9.2 \%$, and $43.8 \pm 4.1 \%$, for the stages I, II and III, respectively), relatively low as a result of the inoculum source used, as well as the salt relative content in industrial effluent. The removals of ammonium, TKN and orthophosphate were between $60-95 \%, 25-37 \%$, and 6-25\%, respectively. The treated effluent requires a post-treatment to reduce organic matter contents and nutrients to the discharge limits allowed in the Republic of Ecuador.

Keywords: Anaerobic sludge, anaerobic treatability, batch reactor, biodegradable matter, industrial effluent.

\section{INTRODUCCIÓN}

La ciudad de Manta constituye el primer puerto pesquero del Ecuador y es considerada como la capital del atún en la costa del Pacífico oriental. Su estratégica ubicación en la zona costera desencadenó el asentamiento de numerosas industrias pesqueras a lo largo de los años, lo cual ha generado importantes problemas, particularmente de índole ambiental. Esto se debe a que la mayoría de estas empresas no cuenta con sistemas propios de tratamiento de 
aguas residuales y opta por evacuarlas, en unos casos, al sistema de alcantarillado doméstico, en otros, a las quebradas y ríos más cercanos a sus instalaciones, o en su defecto, al mar, a través de conexiones directas, sin atender la normativa ambiental vigente [1].

Entre las consecuencias generadas en la zona de influencia de estas empresas se tienen las siguientes: i) contaminación de ríos (Manta y Burro) y áreas costeras, ii) problemas de insalubridad en las zonas de descarga, iii) contaminación atmosférica por malos olores, iv) pérdida del potencial turístico de la región (Playas La Poza, Tarqui, Los Esteros, El Murciélago, otras), v) incumplimiento de la normativa legal vigente en materia de vertido y disposición de efluentes, y vi) colapso del sistema de drenaje urbano y pérdida de eficiencia de la planta de tratamiento local.

La situación antes descritajustifica el desarrollo y evaluación de tecnologías de tratamiento encaminadas a reducir los impactos ambientales ocasionados por el inadecuado manejo y disposición de efluentes industriales, considerando su composición y efectos sobre los ecosistemas naturales. En este sentido, el tratamiento biológico surge como alternativa viable, ya que utiliza la versatilidad metabólica de los microorganismos para la oxidación de la materia orgánica presente en los efluentes (fuente de carbono y/o energía), con la subsecuente reducción del contenido de nutrientes y otros contaminantes [2-5].

Enel tratamientoanaeróbico, loscontaminantes orgánicos (expresados como demanda química de oxígeno, DQO, y/o como demanda bioquímica de oxígeno, $\mathrm{DBO}_{5.20}$ ) son convertidos en lodo biológico y biogás (metano y dióxido de carbono), y queda un pequeño remanente que no es susceptible a la biodegradación.
Las principales ventajas de esta tecnología, especialmente para plantas de tratamiento de grandes dimensiones, son: i) bajos costos de operación, ii) poco requerimiento de espacio, iii) producción de biogás (posible fuente de energía), y iv) baja producción de lodos. De esta manera, los sistemas anaerobios resultan muy adecuados para el tratamiento de aguas residuales de industrias procesadoras de pescado, debido a su alta capacidad de eliminación de materia orgánica. Con ellos es posible conseguir una reducción significativa de los costos de inversión, en comparación a los sistemas aeróbicos, y se generaría además, una cantidad más pequeña de lodo altamente estabilizado y con mayor tendencia a la deshidratación [3, 6-7].

El objetivo de este trabajo consistió en evaluar el tratamiento de las aguas residuales de una industria procesadora de pescado de la ciudad de Manta (Ecuador), en reactores anaeróbicos discontinuos, y establecer su adecuación a las normas ambientales vigentes en materia de vertido al mar y al sistema de alcantarillado.

\section{MATERIALES Y MÉTODOS}

A continuación se describen los materiales y métodos empleados en la presente investigación para evaluar el tratamiento de las aguas residuales de una industria procesadora de pescado de la ciudad de Manta (Ecuador), en reactores anaeróbicos discontinuos, bajo condiciones de laboratorio.

\subsection{EFLUENTE INDUSTRIAL}

El efluente industrial se obtuvo de una empresa procesadora de pescado de la ciudad de Manta (Ecuador), la cual maneja unas 20 
toneladas diarias de atún para la fabricación de conservas (atún en aceite vegetal) y empaques herméticos (sachet y lomos de atún). Dicho efluente correspondió a la mezcla de los residuos líquidos de todos los procesos de la empresa (descongelamiento, limpieza, cocción, envasado, esterilización, etc.), los cuales son almacenados en un tanque de estabilización antes de su incorporación al sistema de alcantarillado público. Las muestras fueron recogidas en recipientes plásticos de $20 \mathrm{~L}$ y transportadas al laboratorio, donde se mantuvieron a $4^{\circ} \mathrm{C}$ hasta su tratamiento.

\subsection{REACTORES ANAERÓBICOS DISCONTINUOS}

Se conformaron cuatro reactores en matraces Erlenmeyer de $1 \mathrm{~L}$, con un contenido de $20 \%$ de lodo biológico anaerobio y $80 \%$ de efluente en tratamiento (Figura 1). El lodo fue obtenido del sedimento de unas lagunas anaeróbicas de la localidad, que eran alimentadas con aguas residuales domésticas. Dicho lodo fue aclimatado con los efluentes durante dos semanas antes de iniciar las pruebas.

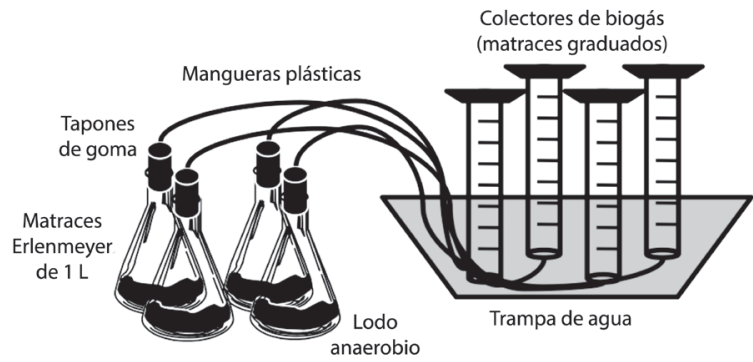

Figura 1. Conformación de los reactores anaeróbicos discontinuos usados en la presente experimentación.

Dos reactores se utilizaron para el tratamiento de los efluentes industriales, mientras que los dos restantes sirvieron como controles, los cuales fueron alimentados con un efluente sintético (Tabla 1). Estos reactores control sirvieron como referencia de comparación para la biodegradabilidad anaeróbica del efluente industrial, ya que contenía glucosa como única fuente de carbono y energía [8].

Tabla 1. Proporciones de nutrientes empleadas para el efluente sintético usado en el presente estudio.

\begin{tabular}{|c|c|}
\hline Compuesto & Cantidad \\
\hline $\mathrm{MgCl}_{2} \cdot 6 \mathrm{H}_{2} \mathrm{O}$ & $0,085 \mathrm{~g} / \mathrm{L}$ \\
\hline$\left(\mathrm{NH}_{4}\right)_{6} \mathrm{Mo}_{7} \mathrm{O}_{24} \cdot 4 \mathrm{H}_{2} \mathrm{O}$ & $40 \mu \mathrm{g} / \mathrm{L}$ \\
\hline $\mathrm{NiSO}_{4} \cdot 6 \mathrm{H}_{2} \mathrm{O}$ & $500 \mu \mathrm{g} / \mathrm{L}$ \\
\hline $\mathrm{CoCl}_{2} \cdot 6 \mathrm{H}_{2} \mathrm{O}$ & $50 \mu \mathrm{g} / \mathrm{L}$ \\
\hline $\mathrm{FeCl}_{2} \cdot 6 \mathrm{H}_{2} \mathrm{O}$ & $0,64 \mu \mathrm{g} / \mathrm{L}$ \\
\hline $\mathrm{H}_{3} \mathrm{BO}_{3}$ & $100 \mu \mathrm{g} / \mathrm{L}$ \\
\hline $\mathrm{NH}_{4} \mathrm{Cl}$ & Variable \\
\hline $\mathrm{CaCl}_{2}$ & $0,04 \mathrm{~g} / \mathrm{L}$ \\
\hline $\mathrm{MnCl}_{2} \cdot 4 \mathrm{H}_{2} \mathrm{O}$ & $500 \mu \mathrm{g} / \mathrm{L}$ \\
\hline $\mathrm{KH}_{2} \mathrm{PO}_{4}+\mathrm{K}_{2} \mathrm{HPO}_{4}$ & Variable \\
\hline $\mathrm{ZnSO}_{4} \cdot 7 \mathrm{H}_{2} \mathrm{O}$ & $500 \mu \mathrm{g} / \mathrm{L}$ \\
\hline$\left(\mathrm{NH}_{4}\right)_{2} \mathrm{SO}_{4}$ & $0,13 \mathrm{~g} / \mathrm{L}$ \\
\hline $\mathrm{NaHCO}_{3}$ & $1,0 \mathrm{~g} / \mathrm{L}$ \\
\hline $\mathrm{CuSO}_{4} \cdot 5 \mathrm{H}_{2} \mathrm{O}$ & $5 \mu \mathrm{g} / \mathrm{L}$ \\
\hline $\mathrm{Fuent} \mathrm{Ch}$ & $1993[8]$ \\
\hline
\end{tabular}

Fuente: Chacín, 1993 [8].

Todos los reactores fueron cerrados herméticamente y funcionaron de manera discontinua, a un tiempo de retención hidráulico (TRH) de 24 h, bajo condiciones mesofílicas y con agitación manual dos veces al día. 


\subsection{TRATAMIENTO ANAERÓBICO DE EFLUENTE INDUSTRIAL}

La digestión anaeróbica de los efluentes de la industria procesadora de pescado se evaluó en tres etapas (Tabla 2), mediante su dilución con agua destilada, a fin de prevenir los choques orgánicos y el efecto de la salinidad sobre la comunidad microbiana del lodo [9-11]. El contenido de glucosa en los reactores control también fue aumentado en las diferentes etapas, con una proporción de DQO un poco mayor que la del efluente industrial, considerando la naturaleza altamente biodegradable de este compuesto orgánico. Cada etapa experimental se extendió hasta observar una estabilidad en las concentraciones de DQO finales (efluente tratado), lo cual se logró aproximadamente a los 25 días de experimentación.

Tabla 2. Etapas de experimentación en los reactores anaeróbicos discontinuos para el tratamiento de los efluentes de la industria procesadora de pescado.

\begin{tabular}{|c|c|c|c|}
\hline \multirow{2}{*}{ Etapa } & \multirow{2}{*}{$\begin{array}{c}\text { Duración } \\
\text { (días) }\end{array}$} & $\begin{array}{c}\text { Tratamiento } \\
\text { Proporción } \\
\text { de efluente } \\
\text { industrial (\%) }\end{array}$ & $\begin{array}{c}\text { Control } \\
\text { DQO teórica } \\
\text { del afluente } \\
\text { (mglucosa)/L) }\end{array}$ \\
\hline I & 25 & 33 & 1000 \\
\hline II & 26 & 66 & 2500 \\
\hline III & 24 & 100 & 5000 \\
\hline
\end{tabular}

Transcurrido el TRH (24 h), los reactores eran descargados usando una manguera a manera de sifón y cargados nuevamente con una porción fresca de efluente industrial. El efluente tratado se usaba para realizar los análisis fisicoquímicos correspondientes.

\subsection{CONTROL Y ANÁLISIS DEL SISTEMA EXPERIMENTAL}

Los parámetros monitoreados para establecer la calidad del efluente final fueron los siguientes, de acuerdo con los métodos estándares: $\mathrm{pH}$, alcalinidad total, $\mathrm{DBO}_{5.20^{\prime}} \mathrm{DQO}$, nitrito, amonio, nitrógeno total Kjeldahl (NTK), ortofosfato, sulfato, sólidos suspendidos totales (SST) y sólidos suspendidos volátiles (SSV) [12]. Todos los análisis fueron realizados por duplicado sobre muestras filtradas (filtros de fibra de vidrio MERCK de $0.7 \mu \mathrm{m}$ de tamaño de poro), con la finalidad de eliminar la interferencia debida a la presencia de sólidos suspendidos. No fue posible cuantificar el volumen de biogás producido, debido a problemas de fuga en las conexiones de los reactores (Figura 1).

\subsection{ANÁLISIS ESTADÍSTICO DE DATOS}

Se calcularon las medias aritméticas y las desviaciones estándares empleando el programa Microsoft Excel 2010 para Windows 7. Se utilizó el programa IBM SPSS Statistics Ver. 20 para realizar un análisis de varianza (ANOVA) de dos vías, con la finalidad de determinar las diferencias significativas en los parámetros fisicoquímicos, considerando el tipo de efluente (tratamiento vs. control) y las etapas experimentales (I, II y III). Antes de realizar el ANOVA se comprobaron, tanto la homogeneidad de las varianzas (Test de Bartlett), como la distribución normal de los residuos (Test de Kolmogorov-Smirnov). Adicionalmente, se estableció un estudio de correlación de Pearson entre los parámetros fisicoquímicos dentro de los reactores para conocer su intervención sobre la remoción de la materia orgánica, considerando $n=75$ y $r_{\text {teórico }}=0.274(p<0.01)$. 


\section{RESULTADOS Y DISCUSIÓN}

En esta sección se describen y discuten los resultados obtenidos durante el tratamiento de las aguas residuales de una industria procesadora de pescado de la ciudad de Manta (Ecuador), en reactores anaeróbicos discontinuos, bajo condiciones de laboratorio.

\subsection{CARACTERIZACIÓN DEL EFLUENTE INDUSTRIAL}

En la Tabla 3 se muestran los resultados de la caracterización fisicoquímica inicial del efluente de la industria procesadora de pescado y su comparación con los límites permisibles de descarga de la República de Ecuador. Estos

Tabla 3. Características fisicoquímicas iniciales del efluente de la industria procesadora de pescado usado en el presente estudio, y límites máximos permisibles para descarga en la República de Ecuador.

\begin{tabular}{|c|c|c|c|}
\hline Parámetro & $X \pm D E$ & $\begin{array}{l}\text { Límite descarga } \\
\text { agua marina* }\end{array}$ & $\begin{array}{l}\text { Límite descarga } \\
\text { alcantarillado* }\end{array}$ \\
\hline $\mathrm{pH}$ & $7.41 \pm 0.52$ & $6-9$ & $5-9$ \\
\hline Alcalinidad total $\left(\mathrm{mgCaCO}_{3} / \mathrm{L}\right)$ & $1105.2 \pm 67.3$ & - & - \\
\hline Conductividad eléctrica (mS/cm) & $9.89 \pm 2.7$ & - & - \\
\hline Potencial redox (mV) & $-14.1 \pm 1.9$ & - & - \\
\hline Oxígeno disuelto (mg/L) & $1.92 \pm 0.33$ & - & - \\
\hline Salinidad (mg/L) & $5730.3 \pm 87.5$ & - & - \\
\hline Cloruro (mg/L) & $1620.7 \pm 84.7$ & - & - \\
\hline $\mathrm{DBO}_{5.20}(\mathrm{mg} / \mathrm{L})$ & $2290.3 \pm 121.7$ & 100 & 250 \\
\hline $\mathrm{DQO}$ (mg/L) & $2565.6 \pm 237.2$ & 250 & 500 \\
\hline Nitrito (mg/L) & $0.39 \pm 0.02$ & - & - \\
\hline Amonio (mg/L) & $79.71 \pm 2.64$ & - & - \\
\hline NTK (mg/L) & $327.08 \pm 5.69$ & 40 & 40 \\
\hline Ortofosfato (mg/L) & $42.67 \pm 1.32$ & 10 (P-total) & 15 (P-total) \\
\hline Aceites y grasas (mg/L) & $6.49 \pm 1.07$ & 0,3 & 100 \\
\hline Sulfato (mg/L) & $1290.60 \pm 54.18$ & - & 400 \\
\hline $\mathrm{SDT}(\mathrm{mg} / \mathrm{L})$ & $5070.4 \pm 93.2$ & - & - \\
\hline SST (mg/L) & $421.6 \pm 64.7$ & 100 & 220 \\
\hline SSV (mg/L) & $360.9 \pm 35.6$ & - & - \\
\hline
\end{tabular}

n=6, X: media aritmética, DE: desviación estándar,

Fuente: República de Ecuador, 2008 [13]. 
resultados reflejan que las concentraciones de $\mathrm{DBO}_{5.20^{\prime}} \mathrm{DQO}, \mathrm{NTK}$, ortofosfato, aceites y grasas, sulfato y SST no cumplen con la norma ambiental [13], lo cual justifica la examinación de tecnologías de tratamiento, dirigidas a minimizar los impactos ambientales ocasionados por el vertido de estos efluentes al ambiente.

La composición fisicoquímica del efluente industrial usado en el presente estudio resultó comparable a la mostrada en otros trabajos, como por ejemplo de Sankpal y Naikwade [14], quienes reportaron una concentración de DQO entre 1200 y $2200 \mathrm{mg} / \mathrm{L}$ para el efluente pesquero de una industria en la India. De igual manera, Cristóvão et al. [4], señalaron un contenido de SST de $284-653 \mathrm{mg} / \mathrm{L}$ y una conductividad eléctrica de $4.0-20.2 \mathrm{mS} / \mathrm{cm}$ para las aguas residuales de una industria de conservas de pescado en el Norte de Portugal.

Por otra parte, el factor de biodegradabilidad (relación $\mathrm{DBO}_{5.20} / \mathrm{DQO}$ ) obtenido para el efluente de la industria procesadora de pescado fue de 0.89 , lo que indica la naturaleza altamente biodegradable del mismo, así como la factibilidad de aplicar tratamiento biológico para remover materia orgánica y nutrientes $[9,15]$.

\subsection{PARÁMETROS FISICOQUÍMICOS}

Las variaciones del $\mathrm{pH}$ y de la alcalinidad total durante el tratamiento del efluente de la industria procesadora de pescado estuvieron dentro de los rangos considerados como óptimos para el crecimiento de microorganismos anaerobios mesófilos [6, 16], los cuales fueron de: $7.13 \pm 0.13,7.62 \pm 0.29$ y $7.61 \pm 0.14 ;$ y de $552.1 \pm 79.7,1025.8 \pm 46.4$ y $1520.8 \pm 96.4 \mathrm{mgCaCO}_{3} / \mathrm{L}$ para las etapas I, II y III, respectivamente. Dichos valores fueron significativamente diferentes $(p<0.0001)$ a los observados en los reactores control y entre las etapas de tratamiento.

Los valores de $\mathrm{pH}$ del efluente industrial tratado estuvieron también dentro del rango establecido por la normativa ecuatoriana para la descarga, tanto en agua marina ( $\mathrm{pH}$ 6-9) como en la red de alcantarillado (pH 5-9) [13]. La norma no contempla límites permisibles de descarga para alcalinidad total.

Por su parte, la concentración inicial de $\mathrm{SO}_{4}^{-2}$ (1290.60 $\pm 54.18 \mathrm{mg} / \mathrm{L}$; Tabla 2) del efluente de procesamiento de pescado se logró disminuir en $44.8 \%, 42.7 \%$ y $32.9 \%$ durante el tratamiento en los reactores anaeróbicos discontinuos (etapas I, II y III, respectivamente). El proceso que domina bajo las condiciones del ensayo establece que el sulfato se reduzca a $\mathrm{H}_{2} \mathrm{~S}$ vía reducción desasimilativa [17-18], mediante el acoplamiento de la oxidación de la materia orgánica (donador de electrones) a la reducción de sulfato (aceptor de electrones) con la participación de bacterias fermentativas hidrolíticas que degradan materia orgánica compleja [19].

Los contenidos de SST y de SSV en el efluente tratado presentaron valores medios de $88.44 \pm 24.44, \quad 79.62 \pm 9.45$ y $104.86 \pm 22.68$ $\mathrm{mg} / \mathrm{L} ;$ y de $69.84 \pm 18.55, \quad 71.58 \pm 9.51$ y $85.72 \pm 13.13 \mathrm{mg} / \mathrm{L}$ para las etapas I, II y III, respectivamente. Las concentraciones de SST estuvieron por debajo de los límites máximos permitidos para el vertido en agua marina (100 $\mathrm{mg} / \mathrm{L}$ ) y en el alcantarillado (220 mg/L) [13], a excepción de las observadas en la etapa III, como resultado del incremento en la carga orgánica de los reactores. Por tal motivo, se hace necesario monitorear minuciosamente el contenido de sólidos en el efluente final para verificar la necesidad de implementar un sistema de sedimentación posterior. 


\subsection{MATERIA ORGÁNICA}

En cuanto al contenido de materia orgánica en el efluente tratado, las concentraciones medias de $\mathrm{DBO}_{5.20}$ estuvieron en $285.36 \pm 7.41$, $710.71 \pm 28.72$ y $908.61 \pm 4.82 \mathrm{mg} / \mathrm{L}$, mientras que las de DQO fueron de $429.77 \pm 61.21$, $1099.48 \pm 163.87$ y $1361.96 \pm 103.70 \mathrm{mg} / \mathrm{L}$ para las etapas I, II y III, respectivamente. Se observaron diferencias altamente significativas $(p<0.0001)$ de estas concentraciones con respecto al tipo de efluente y las etapas experimentales.

La Figura 2 muestra el comportamiento de la DQO durante el tratamiento del efluente resultante del procesamiento de pescado en los reactores anaeróbicos discontinuos. Las concentraciones de DQO en el efluente tratado fueron proporcionales a las de entrada, lo que repercutió sobre los porcentajes de remoción de DQO conforme se aumentaba la relación de efluente industrial desde la etapa I hasta la III. La remoción de materia orgánica, medida como DQO, fue relativamente semejante en los reactores cargados con las aguas residuales industriales y los de control (Tabla 4), y se obtuvieron medias aritméticas de $34.7 \pm 9.7$, $36.9 \pm 9.2$ y $43.8 \pm 4.1 \%$ para el efluente pesquero en las etapas I, II y III, respectivamente.

Los valores finales de DQO correlacionaron significativamente con el pH $(r=0.661, p<0.01)$,
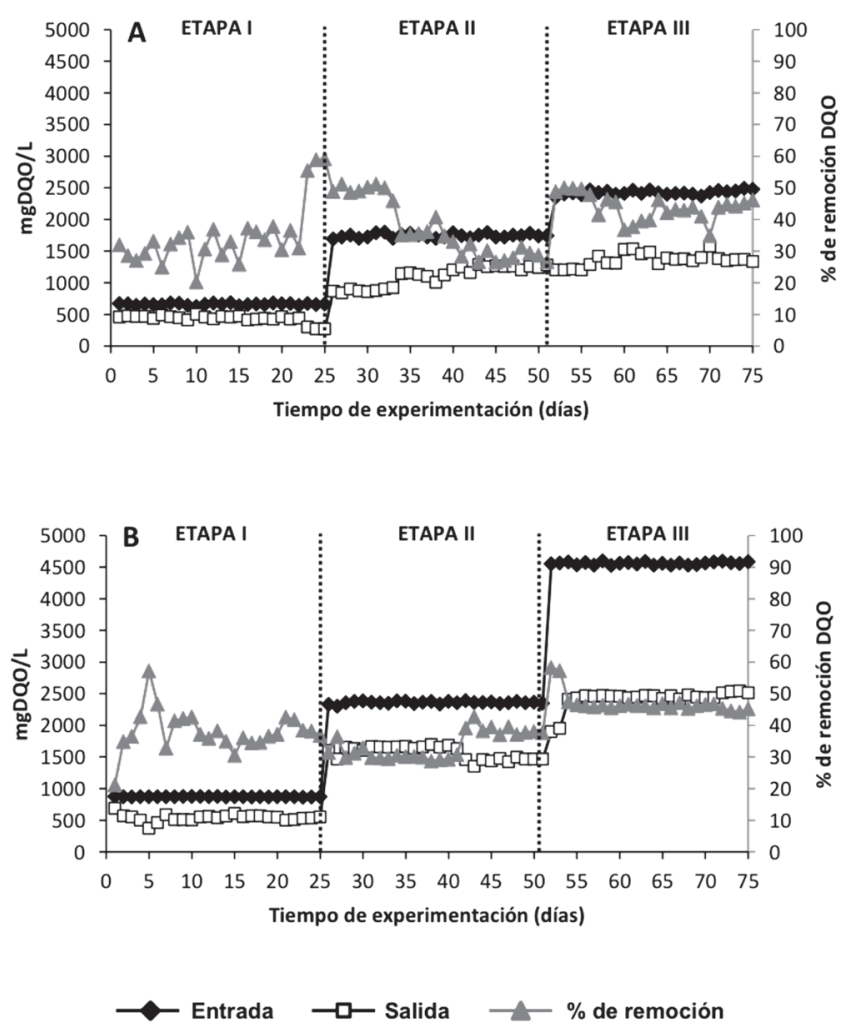

Figura 2. Comportamiento de las concentraciones de demanda química de oxígeno (DQO) durante el tratamiento del efluente resultante del procesamiento de pescado en reactores anaeróbicos discontinuos. A) Efluente de pescado, B) Reactor control. 
Tabla 4. Porcentajes de remoción de materia orgánica y nutrientes durante el tratamiento del efluente resultante del procesamiento de pescado en reactores anaeróbicos discontinuos.

\begin{tabular}{|c|c|c|c|c|c|c|}
\hline Etapa & DBO $_{5.20}$ & DQO & Nitrito & Amonio & NTK & Ortofosfato \\
\hline \multicolumn{7}{|c|}{ Efluente de procesamiento de pescado } \\
\hline$I^{*}$ & $37.9 \pm 4.1$ & $34.7 \pm 9.7$ & - & $60.7 \pm 1.2$ & $24.6 \pm 3.9$ & $6.4 \pm 1.7$ \\
\hline$I^{* *}$ & $41.8 \pm 7.6$ & $36.9 \pm 9.2$ & $61.9 \pm 2.89$ & $5.5 \pm 1.8$ & $28.9 \pm 5.7$ & $10.3 \pm 1.8$ \\
\hline$I^{* * *}$ & $46.2 \pm 3.2$ & $43.8 \pm 4.1$ & $56.3 \pm 3.4$ & $85.4 \pm 0.7$ & $37.2 \pm 1.0$ & $24.8 \pm 2.0$ \\
\hline \multicolumn{7}{|c|}{ Reactor control } \\
\hline$I^{*}$ & $40.2 \pm 2.6$ & $38.0 \pm 6.4$ & - & $97.8 \pm 0.4$ & $95.7 \pm 0.4$ & $7.3 \pm 1.6$ \\
\hline$I^{* *}$ & $37.7 \pm 3.8$ & $33.7 \pm 4.4$ & - & $99.7 \pm 0.1$ & $96.5 \pm 0.5$ & $11.6 \pm 1.1$ \\
\hline$I^{* * *}$ & $50.3 \pm 2.9$ & $47.0 \pm 3.4$ & - & $99.7 \pm 0.2$ & $97.2 \pm 0.3$ & $18.0 \pm 2.1$ \\
\hline
\end{tabular}

alcalinidad total $(r=0.907, p<0.01)$, nitrito $(r=0,754, p<0,01)$, amonio $(r=-0.754, p<0.01)$, NTK $(r=0.964, p<0.01)$, ortofosfato $(r=0.950$, $p<0.01)$, sulfato $(r=0.870, p<0.01)$ y SSV $(r=0.343, p<0.01)$, lo que indicala interacción de estos parámetros fisicoquímicos durante el proceso de biodegradación de la materia orgánica presente en el efluente industrial.

De manera general, los porcentajes de remoción de materia orgánica en los reactores anaeróbicos, expresados como $\mathrm{DBO}_{5.20}$ y DQO, fueron relativamente bajos, por ejemplo, al ser comparados con los reportados por $\mathrm{Li}$ et al. [20], quienes encontraron $79 \%$ de remoción de materia orgánica en un reactor UASB para un efluente sintético salino que contenía 4050 $\mathrm{mgDQO} / \mathrm{L}$. Asimismo, Alexandre et al. [21] hallaron una remoción entre $48.4 \%$ y $82.0 \%$ (expresada como DQO soluble) para el efluente de una industria procesadora de pescado brasileña, sometido previamente a hidrólisis enzimática, cuya DQO inicial era de $6612 \pm 4336$ $\mathrm{mg} / \mathrm{L}$.
Entre los factores que intervienen sobre la eficiencia de remoción de materia orgánica en sistemas de tratamiento biológico, particularmente en procesos anaeróbicos, se pueden mencionar la calidad u origen del inóculo, así como el contenido de sales en el efluente [10-11]. La salinidad puede influir sobre las interacciones osmóticas y electroquímicas de la membrana celular con el medio externo, interviniendo en el flujo de solutos y solventes [11]. Por ejemplo, se ha encontrado inhibición de la metanogénesis a concentraciones de $\mathrm{Na}^{+}$por encima de 5.25 $\mathrm{g} / \mathrm{L}$ durante el tratamiento de efluentes que contienen insulina en biorreactores UASB [22]. Este efecto negativo de la salinidad sobre la eficiencia de biodegradación de materia orgánica en sistemas de tratamiento anaeróbico ha sido contrarrestado mediante la adición de «solutos compatibles» que ayudan a los microorganismos a confrontar dicho efecto [23-24]. De igual forma, la codigestión de aguas residuales de pesquerías con desechos agropecuarios, como corteza de árboles y estiércol de vaca, también ha mostrado ser 
una alternativa viable para mejorar la eficiencia del tratamiento anaeróbico [25].

Con respecto a la fuente y características del inóculo, se ha reportado que las mismas determinan la eficiencia de los sistemas biológicos de tratamiento. Panswad y Anan [26] encontraron una reducción del contenido orgánico de $60 \%$ en un reactor anaeróbico/ anóxico/aeróbico a escala de laboratorio durante el tratamiento de un efluente sintético compuesto por sacarosa y ácido acético como fuentes de carbono $y$, un contenido salino de $30 \mathrm{~g} / \mathrm{L}$, utilizando un inóculo no aclimatado, mientras que dicha reducción aumentó a $71 \%$ cuando incluyeron un inóculo aclimatado. Adicionalmente, Aspé et al. [27], para el tratamiento de aguas residuales de pesquerías, observaron remociones de materia orgánica de hasta $96 \%$ al utilizar sedimentos marinos como fuente de inóculo en reactores anaeróbicos de mezcla completa. Este planteamiento anterior puede explicar la relativa baja eficiencia de los reactores utilizados en el presente estudio en
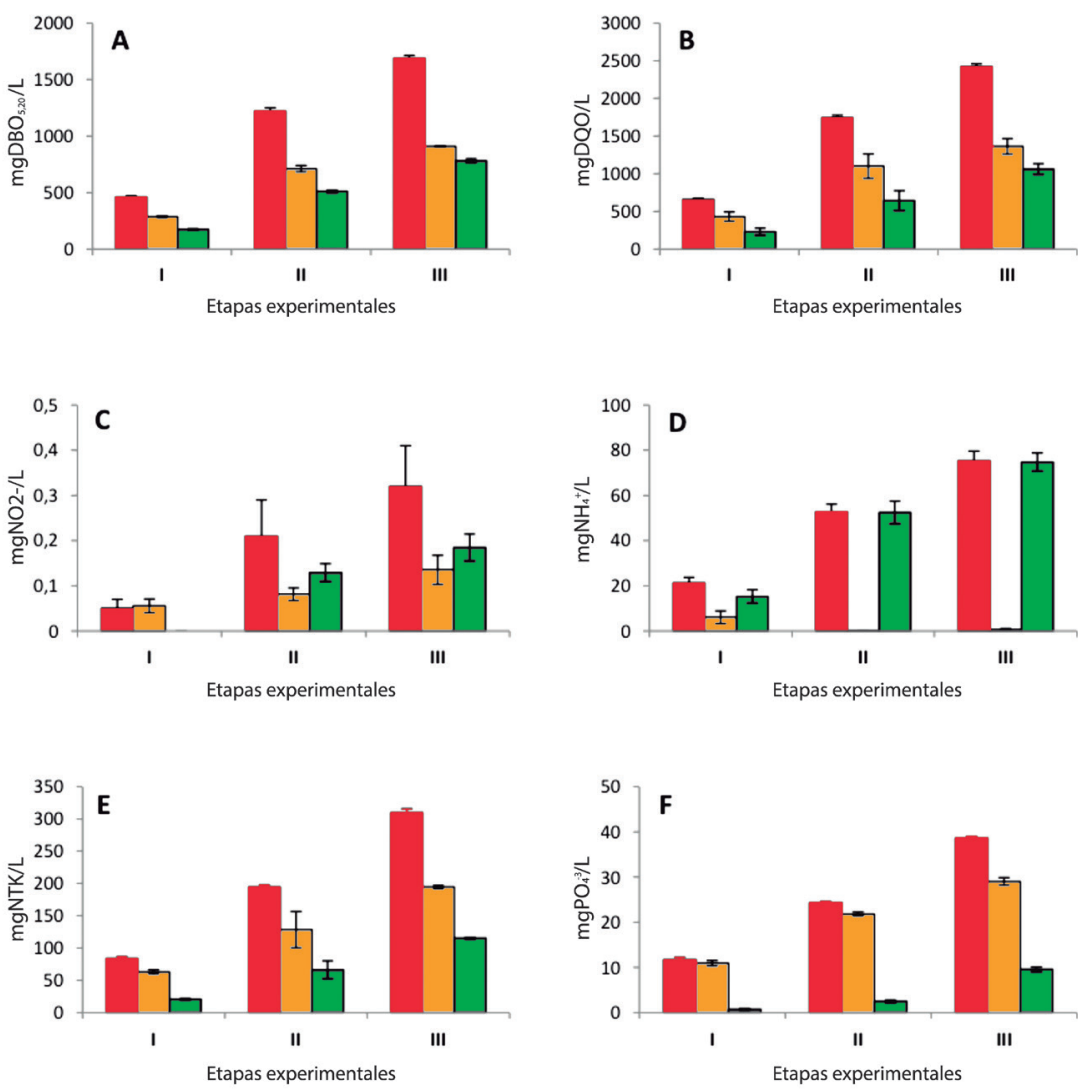

Entrada

Salida

口 Removido

Figura 3. Cantidad de materia orgánica y nutrientes removidos durante el tratamiento del efluente resultante del procesamiento de pescado en reactores anaeróbicos discontinuos. A) DBO, B) DQO, C) Nitrito, D) Amonio, E) NTK y F) Ortofosfato. 
cuanto a remoción de materia orgánica, ya que si bien el lodo fue aclimatado paulatinamente al efluente salino de la industria procesadora de pescado, el mismo procedía de lagunas anaeróbicas que trataban efluentes alimenticios no salinos, lo cual no garantiza la presencia de una microbiota halófila dominante.

La cantidad de materia orgánica removida durante el tratamiento de efluente industrial en los reactores anaeróbicos discontinuos, medida como $\mathrm{DBO}_{5.20}$ (Figura 3A) y DQO (Figura $3 B)$, fue de 174.85 $\pm 4.69,509.66 \pm 12.00 \mathrm{y}$ $781.80 \pm 16.94 \mathrm{mg} / \mathrm{L}$ y de 229.32 \pm 46.36 , $642.18 \pm 131.61$ y $1061.46 \pm 69.55 \mathrm{mg} / \mathrm{L}$, respetivamente, para las etapas I, II y III. En la
Figura 3B puede observarse que, considerando la concentración de DQO de entrada $(659.09 \pm 14.85 \mathrm{mg} / \mathrm{L})$ y salida $(429.77 \pm 61.21$ $\mathrm{mg} / \mathrm{L})$, solo el efluente final de la etapa I, cumplió con el límite permitido en la norma ecuatoriana para la descarga al sistema de alcantarillado (500 mgDQO/L) [13].

\subsection{NITRÓGENO Y FÓSFORO}

La remoción de nitrito durante el tratamiento del efluente industrial (etapa II de 61.9 $\pm 2.8 \%$ y etapa III de $56.3 \pm 3.4 \%$, Tabla 4) fue significativamente diferente $(p<0.0001)$ con respecto al tipo de efluente (glucosa frente a efluente de pescado) y las etapas
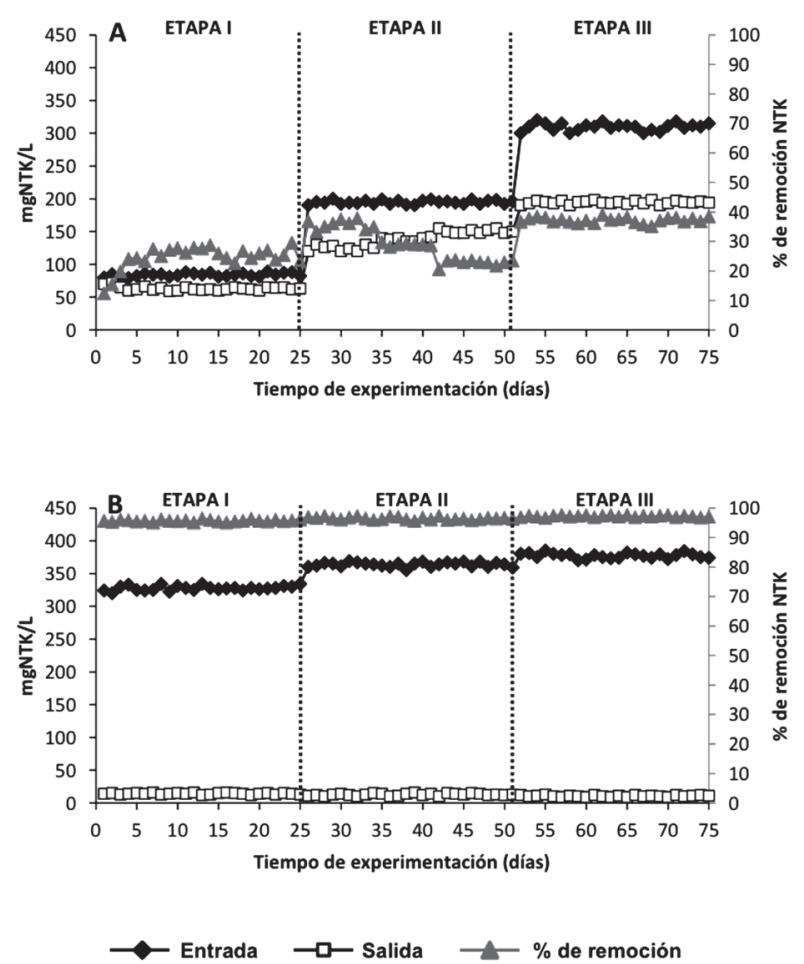

Figura 4. Comportamiento de las concentraciones de nitrógeno total Kjeldahl (NTK) durante el tratamiento del efluente resultante del procesamiento de pescado en reactores anaeróbicos discontinuos. A) Efluente de pescado, B) Reactor control. 
de tratamiento, como resultado de su concentración inicial $(0.21 \pm 0.06$ y $0.32 \pm 0.08$ $\mathrm{mgNO}_{2}^{-} / \mathrm{L}$, respectivamente; Figura $3 \mathrm{C}$ ) y de las condiciones de reducción establecidas. Por su parte, el contenido de $\mathrm{NH}_{4}{ }^{+}$se pudo reducir por encima del $60 \%$ tanto en los reactores alimentados con efluente industrial como en los controles (Tabla 4) y se obtuvieron concentraciones finales de $6.04 \pm 2.75$, $0.25 \pm 0.03$ y $0.61 \pm 0.37 \mathrm{mgNH}_{4}^{+} / \mathrm{L}$ y de $\mathrm{NH}_{4}^{+}$ removido de $15.27 \pm 3.02,52.45 \pm 5.33$ y $74.72 \pm 4.21 \mathrm{mg} / \mathrm{L}$ (Figura 3D) para las aguas residuales de la industria procesadora de pescado en las etapas I, II y III, respectivamente. Las concentraciones de NTK exhibieron cierta variabilidad con relación a la proporción de efluente aplicada (Figura 4), siendo significativamente diferentes $(p<0.0001)$ con respecto al tipo de efluente y las etapas experimentales. Los valores medios finales de NTK $(63.12 \pm 3.31,128.43 \pm 28.08$ y $194.38 \pm 2.41 \mathrm{mg} / \mathrm{L}$ para las etapas I, II y III, respectivamente) no cumplieron con el límite de descarga establecido por la legislación ecuatoriana (40 mgNTK/L) [13], debido a los porcentajes de remoción obtenidos: $24.6 \pm 3.9 \%, 28.9 \pm 5.7 \%$ y $37.2 \pm 1,0 \%$, durante las etapas I, II y III, respectivamente (Tabla 4). La Figura 3E muestra la cantidad de NTK removido durante cada etapa de tratamiento.
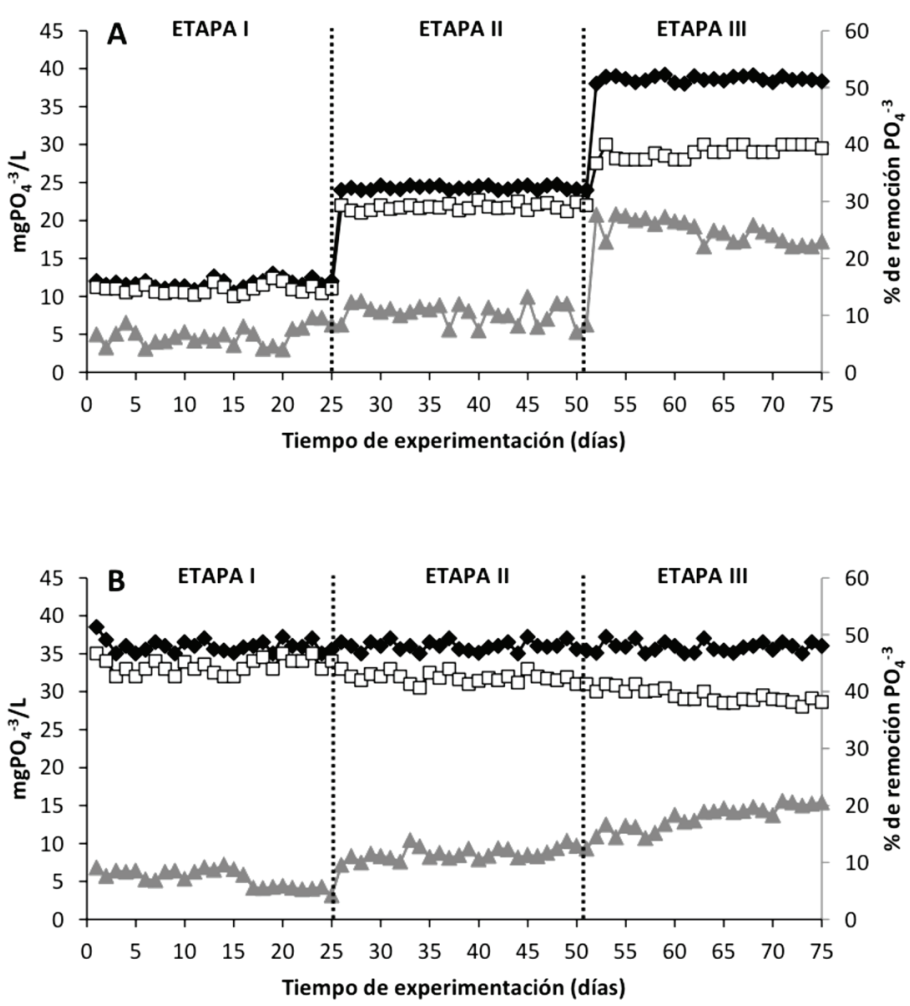

$\multimap$ Entrada $\longrightarrow$ Salida $\longrightarrow$ \% de remoción

Figura 5. Comportamiento de las concentraciones de ortofosfato durante el tratamiento del efluente resultante del procesamiento de pescado en reactores anaeróbicos discontinuos. A) Efluente de pescado, B) Reactor control. 
Los procesos que determinan las variaciones de las formas de nitrógeno en los reactores biológicos son básicamente de naturaleza microbiana. La desnitrificación es un proceso respiratorio anaerobio heterotrófico, a través del cual ocurre la reducción de $\mathrm{NO}_{3}{ }^{-}$hasta $\mathrm{N}_{2}$ en una serie de etapas y con la intervención de la actividad de diferentes enzimas. El proceso general de reducción podría establecerse de la siguiente manera: $\mathrm{NO}_{3}{ }^{-} \rightarrow \mathrm{NO}_{2}{ }^{-} \rightarrow \mathrm{NO} \rightarrow \mathrm{N}_{2} \mathrm{O}$ $\rightarrow \mathrm{N}_{2^{\prime}}$ donde cada reacción es mediada por un grupo especialista de microorganismos, entre los que se encuentran Alcaligenes, Paracoccus, Pseudomonas, Thiobacillus y Thiosphaera, entre otros [28-29]. De esta manera, y bajo las condiciones anaeróbicas establecidas en los reactores discontinuos del presente estudio, se pudieron reducir las concentraciones iniciales de $\mathrm{NO}_{2}^{-}$a $\mathrm{N}_{2} \mathrm{O}$ a algún otro producto intermediario, como $\mathrm{NO} \circ \mathrm{N}_{2} \mathrm{O}$.

También se ha reportado la remoción de amonio en efluentes con una baja relación $\mathrm{C} / \mathrm{N}$, como los de industrias de conservas de pescado[30], mediante la oxidación anaeróbica de $\mathrm{NH}_{4}{ }^{+}$y usando $\mathrm{NO}_{2}{ }^{-}$como aceptor final de electrones (proceso conocido como ANAMMOX, por sus siglas en inglés), para generar $\mathrm{N}_{2}$ por acción de bacterias quimiolitoautotrofas (Ej. Planctomycetes) [31]. Dapena-Mora et al [30] encontraron remociones de $\mathrm{N}$ entre $35 \%$ y $90 \%$ (considerando las concentraciones de $\mathrm{NH}_{4}{ }^{+}$, $\mathrm{NO}_{2}^{-}$y $\mathrm{NO}_{3}^{-}$) vía ANAMMOX para efluentes de una fábrica de conservas de pescado en un reactor por carga secuencial (SBR, según sus siglas en inglés), las cuales resultan comparables a las obtenidas en el presente estudio para las distintas formas de N (Tabla 4).

Las concentraciones de ortofosfato en el efluente tratado fueron dependientes de las del influente (entrada) (Figura 5), al igual que lo observado para la materia orgánica (Figura
2), y se obtuvo mayor remoción a medida que se aumentó la proporción de efluente industrial entre las etapas experimentales. Dicho comportamiento fue significativamente diferente $(p<0.0001)$ considerando el tipo de efluente y las etapas de tratamiento. De esta manera, los porcentajes de remoción de $\mathrm{PO}_{4}^{-}$ ${ }^{3}$ para las aguas residuales de pescado fueron de $6.4 \pm 1.7 \%, 10.3 \pm 1.8 \%$ y $24.8 \pm 2.0 \%$ (Tabla 4 ), para concentraciones finales de $10.93 \pm 0.59$, $21.78 \pm 0.42$ y $29.02 \pm 0.85 \mathrm{mg} / \mathrm{L}$ (Figura 3F) durante las etapas I, II y III, respectivamente, las cuales superaron el límite de descarga (10 mg/L como P-total para agua marina) contemplado en la normativa ambiental ecuatoriana [13].

En la Figura $3 \mathrm{~F}$ se observan las cantidades de $\mathrm{PO}_{4}^{-3}$ removidas durante el tratamiento anaeróbico del efluente del procesamiento de pescado en reactores discontinuos, que fueron de $0.75 \pm 0.20 \mathrm{mg} / \mathrm{L}$ para la etapa l; de $2.51 \pm 0.33 \mathrm{mg} / \mathrm{L}$ para la etapa II y de $9.58 \pm 0.49 \mathrm{mg} / \mathrm{L}$ para la etapa III. Panswad y Anan [26] indicaron que la eliminación biológica convencional de $P$ es sensible a las condiciones osmóticas y se reduce rápidamente con el aumento de la salinidad, incluso cuando el lodo ha sido aclimatado previamente. En este sentido, Hong et al. [32] observaron una reducción de $20 \%$ en la eficiencia de remoción de $\mathrm{P}$ a partir de una concentración de cloruro de 1500 mg/L e inhibición completa de la remoción a 2500 $\mathrm{mgCl} / \mathrm{L}$ durante su experimentación con efluente sintético en un reactor anaeróbico/ anóxico/aeróbico. Dicho hallazgo podría explicar los relativos bajos porcentajes de remoción de materia orgánica y ortofosfato obtenidos en el presente estudio para los ensayos con el efluente de la industria procesadora de pescado. 


\section{CONCLUSIONES}

El tratamiento de las aguas residuales de la industria procesadora de pescado de la ciudad de Manta (Ecuador) en reactores anaeróbicos discontinuos mostró una relativa baja eficiencia, posiblemente como resultado del lodo inóculo usado, así como del contenido relativo de sales. El efluente tratado requiriere de la aplicación de un postratamiento, para reducir el contenido de materia orgánica y nutrientes a los límites permisibles de descarga establecidos en la República de Ecuador. Se recomienda realizar ensayos de biodegradabilidad con microorganismos aerobios, para comparar la eficiencia de los tratamientos.

Se observaron indicios de la ocurrencia de la oxidación anaeróbica de $\mathrm{NH}_{4}{ }^{+}$usando $\mathrm{NO}_{2}{ }^{-}$ como aceptor final de electrones (proceso conocido como ANAMMOX, por sus siglas en inglés), con porcentajes de remoción de $\mathrm{NH}_{4}{ }^{+} \mathrm{y}$ $\mathrm{NO}_{2}{ }^{-}$significativamente altos.

\section{AGRADECIMIENTOS}

A la Secretaría Nacional de Educación Superior, Ciencia, Tecnología e Innovación de Ecuador (SENESCYT); mediante el Proyecto PROMETEO, la Secretaría Nacional de Planificación y Desarrollo de Ecuador (SENPLADES) y la Universidad Laica "Eloy Alfaro" de Manabí (ULEAM). Los autores desean expresar su agradecimiento al Centro de Servicio para el Control de la Calidad (CESECCA) de la Facultad de Ingeniería Industrial-ULEAM, así como a los estudiantes C. Chiriboga, J. Meza y J. Roldán de la ULEAM, por su colaboración durante los ensayos de laboratorio.

\section{REFERENCIAS BIBLIOGRÁFICAS}

[1] Lynch, G. (2007). Auditoría ambiental al proyecto de control de la contaminación del río Manta y su área de influencia de la empresa de agua potable y alcantarillado de Manta, EAPAM. Manta, Ecuador: Dirección de Auditoría de Proyectos y Ambiente de Manta. p. 71.

[2] Chan, Y.J., Chong, M.F., Law, C.L. y Hassell, D.G., (2009). A review on anaerobicaerobic treatment of industrial and municipal wastewater. Chemical Engineering Journal, 155, 1-18.

[3] Chowdhury, P., Viraraghavan, T. y Srinivasan, A. (2010). Biological treatment processes for fish processing wastewater - A review. Bioresource Technology, 101, 439-449.

[4] Cristóvão, R.O., Botelho, C.M.S., Martins, R.J.E, y Boaventura, R.A.R. (2012). Chemical and biological treatment of fish canning wastewaters. International Journal of Bioscience, Biochemistry and Bioinformatics, 2, 237-242.

[5] Muthukumaran, S. y Baskaran, K. (2013). Organic and nutrient reduction in a fish processing facility - A case study. International Biodeterioration \& Biodegradation, 85, 563-570.

[6] Latif, M.A., Ghufran, R., Wahid, Z.A. y Ahmad, A. (2011). Integrated application of upflow anaerobic sludge blanket reactor for the treatment of wastewaters. Water Research, 45, 4683-4699.

[7] Nges, I.A., Mbatia, B. y Björnsson, L. (2012). Improved utilization of fish waste by anaerobic digestion following 
omega-3 fatty acids extraction. Journal of Environmental Management, 110, 159-165.

[8] Chacín, E. (1993). Treatment characteristics of two phase anaerobic system using an UASB reactor (Tesis doctoral inédita). University of Birmingham, Birmingham Inglaterra.

[9] Aloui, F., Khoufi, S., Loukil, S. y Sayadi, S. (2009). Performances of an activated sludge process for the treatment of fish processing saline wastewater. Desalination, 246, 389-396.

[10] Xiao, Y. y Roberts, D.J. (2010). A review of anaerobic treatment of saline wastewater. Environmental Technology, 31, 1025-1043.

[11] Yang, J., Spanjers, H., Jeison, D. y van Lier, J.B., (2013). Impact of $\mathrm{Na}+$ on biological wastewater treatment and the potential of anaerobic membrane bioreactors: A review. Critical Reviews in Environmental Science and Technology, 43, 2722-2746.

[12] American Public Health Association (APHA), American Water Works Association (AWWA) y Water Environment Federation (WEF). (2005). Standard Methods for the Examination of Water and Wastewater (21th Edition). Washington, D.C. EE.UU.: American Public Health Association.

[13] República de Ecuador. (2008). Libro VI, Norma de calidad ambiental y de descarga de efluentes: recurso agua. Anexo 1 (pp.286-340). Ecuador: Presidencia de la República de Ecuador.
[14] Sankpal, S.T. y Naikwade, P.V. (2012). Physicochemical analysis of effluent discharge of fish processing industries in Ratnagiri India. Bioscience Discovery, 3(1), 107-111.

[15] Tay, J.H., Show, K.Y. y Hung, Y.T. (2006). Seafood processing wastewater treatment. En L.K. Wang, Y.T. Hung, H.H. Lo y C. Yapijakis (Eds.), Waste Treatment in the Food Processing Industry (pp.29-66). Boca Ratón, FL, EE.UU.: Taylor \& Francis Group.

[16] Sandberg, M. y Ahring, B.K. (1992). Anaerobic treatment of fish meal process waste-water in a UASB reactor at high $\mathrm{pH}$. Applied Microbiology and Biotechnology, 36, 800-804.

[17] Bai, H., Yang, Y., Quan, H., Han, Y., Sun, J. y Feng, Y. (2013). Bioremediation of copper-containing wastewater by sulfate reducing bacteria coupled with iron. Journal of Environmental Management, 129, 350-356.

[18] Sarti, A. y Zaiat, M. (2011). Anaerobic treatment of sulfate-rich wastewater in an anaerobic sequential batch reactor (AnSBR) using butanol as the carbon source. Journal of Environmental Management, 92, 1537-1541.

[19] Celis-García, L.B., Villa-Gómez, D., Alpuche-Solís, A.G., OrtegaMorales, B.O. y Razo-Flores, E. (2009). Characterization of sulfatereducing bacteria dominated surface communities during start-up of a down-flow fluidized bed reactor. Journal of Industrial Microbiology and Biotechnology, 36, 111-121. 
[20] Li, J., Yu, L., Yu, D., Wang, D., Zhang, P. y Ji, Z. (2014). Performance and granulation in an upflow anaerobic sludge blanket (UASB) reactor treating saline sulfate wastewater. Biodegradation, 24(1), pp. 127-136.

[21] Alexandre, V.M.F., Valente, A.M., Cammarota, M.C. y Freire, D.M.G. (2011). Performance of anaerobic bioreactor treating fish-processing plant wastewater pre-hydrolyzed with a solid enzyme pool. Renewable Energy, 36, 3439-3444.

[22] Boardman, G.D., Tisinger J.L., y Gallagher D.L. (1995). Treatment of clam processing wastewaters by means of upflow anaerobic sludge blank technology. Water Research, 29, 1483.

[23] Vyrides I y Stuckey, D.C. (2009). Adaptation of anaerobic biomass to saline conditions: Role of compatible solutes and extracellular polysaccharides. Enzyme and Microbial Technology, 44, 46-51.

[24] Yerkes, D.W., Boonyakitsombut, S. y Speece R.E. (1997). Antagonism of sodium toxicity by the compatible solute betaine in anaerobic methanogenic systems. Water Science and Technology, 36, 15-24.

[25] Estevez, M.M., Sapci, Z., Linjordet, R. y Morken, J. (2014). Incorporation of fish by-product into the semicontinuous anaerobic co-digestion of pre-treated lignocellulose and cow manure, with recovery of digestate's nutrients. Renewable Energy, 66, 550-558.
[26] Panswad, T. y Anan, C. (1999). Impact of high chloride wastewater on an anaerobic/anoxic/aerobic process with and without inoculation of chloride acclimated seeds. Water Research, 33, 1165-1172.

[27] Aspé, E., Martí, M.C., Jara, A. y Roeckel M. (2001). Ammonia inhibition in the anaerobic treatment of fishery effluents. Water Environment Research, 73, 154-164.

[28] Andalib, M., Nakhla, G., McIntee, E. y Zhu J. (2011). Simultaneous denitrification and methanogenesis (SDM): Review of two decades of research. Desalination, 279, 1-14.

[29] Cervantes-Carrillo, F., Pérez, J. y Gómez, J. (2000). Avances en la eliminación biológica del nitrógeno de las aguas residuales. Revista Latinoamericana de Microbiología, 42, 73-82.

[30] Dapena-Mora, A., Campos, J.L., Mosquera-Corral, A. y Méndez, R. (2006). Anammox process for nitrogen removal from anaerobically digested fish canning effluents. Water Science and Technology, 53(12), 265-274.

[31] Magrí, A., Béline, F. y Dabert, P. (2013). Feasibility and interest of the anammox process as treatment alternative for anaerobic digester supernatants in manure processing - An overview. Journal of Environmental Management, 131, 170-184.

[32] Hong, C.C., Chan, S.K. y Shim, H. (2007). Effect of chloride on biological nutrient removal from wastewater. Journal of Applied Sciences in Environmental Sanitation, 2(3), 85-92. 